\title{
The role of serum growth hormone and insulin-like growth factor-1 in adult humans brain morphology
}

\author{
Taoyang Yuan ${ }^{1}$, Jianyou Ying ${ }^{1}$, Lu Jin ${ }^{2}$, Chuzhong Li ${ }^{1}$, Songbai Gui ${ }^{2}$, Zhenye Li ${ }^{2}$, Rui Wang ${ }^{1}$, \\ Zhentao Zuo ${ }^{3,4,5}$, Yazhuo Zhang ${ }^{1,2,6}$ \\ ${ }^{1}$ Beijing Neurosurgical Institute, Capital Medical University, Beijing, China \\ ${ }^{2}$ Department of Neurosurgery, Beijing Tiantan Hospital, Capital Medical University, Beijing, China \\ ${ }^{3}$ State Key Laboratory of Brain and Cognitive Science, Institute of Biophysics, Chinese Academy of Sciences, \\ Beijing, China \\ ${ }^{4}$ CAS Center for Excellence in Brain Science and Intelligence Technology, Chinese Academy of Sciences, Beijing, China \\ ${ }^{5}$ Sino-Danish College, University of Chinese Academy of Sciences, Beijing, China \\ ${ }^{6}$ Beijing Institute for Brain Disorders, Brain Tumour Center, China National Clinical Research Center for \\ Neurological Diseases, Key Laboratory of Central Nervous System Injury Research, Beijing, China
}

Correspondence to: Zhentao Zuo, Yazhuo Zhang; email: ztzuo@bcslab.ibp.ac.cn, zyz2004520@yeah.net

Keywords: growth hormone (GH), insulin-like growth factor-1 (IGF-1), brain structure, structural magnetic resonance imaging analysis

Received: October 14, 2019 Accepted: December 25, 2019 Published: January 22, 2020

Correction: This article has been corrected. Please see Aging 2021: https://doi.org/10.18632/aging.203601

Copyright: Yuan et al. This is an open-access article distributed under the terms of the Creative Commons Attribution License (CC BY 3.0), which permits unrestricted use, distribution, and reproduction in any medium, provided the original author and source are credited.

\begin{abstract}
Growth hormone (GH) and its anabolic mediator, insulin-like growth factor-1 (IGF-1), have a critical role in the central nervous system. However, their detailed roles in the adult human brain are not clear. In this study, structural MRIs of 48 patients with GH-secreting pituitary adenoma (GH-PA), 48 sex- and age-matched clinical Non-Functional pituitary adenoma patients (NonFun-PA) and healthy controls (HCs) were assessed using voxelbased morphometry (VBM) and region-based morphometry (RBM). Correlation analyses helped determine the relationships between serum hormone levels and brain structure. The whole-brain gray matter volume (GMV) and white matter volume (WMV) significantly increased at the expense of cerebrospinal fluid volume (CSFV) in GH-PA (Bonferroni corrected, $p<0.01$ ). The increase in GMV and reduction in CSFV were significantly correlated with serum GH/IGF-1 levels ( $p<0.05$ ). VBM showed significant correlations of the GMV/WMV alteration pattern between GH-PA vs HCs and GH-PA vs NonFun-PA and widespread bilateral clusters of significantly increased GMV and WMV in GH-PA (pFDR<0.05). RBM showed obviously increased GMV/WMV in 54 of 68 brain regions $(p<0.05)$ in GH-PA compared to HCs. Our results provide imaging evidence that serum GH/IGF-1 contributes to brain growth, which may be a potential treatment option for neurodegenerative disorders and brain injury in humans.
\end{abstract}

\section{INTRODUCTION}

Growth hormone (GH) is secreted by somatotrophs within the anterior pituitary gland and leads to the production of insulin-like growth factor (IGF-1) in the liver [1]. GH and IGF-1 signaling are critical for the proper development and function of many major organ systems by regulating the growth of somatic cells, especially in early development and during adolescence
$[2,3]$. Previous animal studies reported robust expression of GH and IGF-1 receptors in the central nervous system (CNS), including the cerebral cortex, thalamus, hypothalamus, cerebellum, brainstem, and hippocampus $[4,5]$. Meanwhile, GH and IGF-1 in circulation have been shown to cross the blood-brain barrier into the brain tissue and cerebrospinal fluid (CSF) to activate GH/IGF1 receptors [3, 6-8]. The potential roles of $\mathrm{GH}$ and IGF-1 in brain growth, development, neurogenesis, and 
neuroprotection, as well as their functional roles in behavior, cognition and neurotransmission, have been investigated in vivo and in vitro [9-12]. For instance, GH or IGF-1 gene knock-out mice, compared to control animals, exhibited CNS deficits including reduced brain size, loss of myelination and specific parvalbumincontaining neurons, and cognitive impairments $[13,14]$. Long-term GH/IGF-1 replacement has been shown to improve learning and memory in aged rats [15-17]. In animal models of neurodegenerative diseases, GH/IGF-1 administration improved cognition function [18]. Furthermore, exogenous GH/IGF-1 administration facilitated the proliferation, differentiation, survival, and migration of neural stem/precursor cells after brain injury in animals $[19,20]$. In humans, some studies have suggested that GH/IGF-1 treatment can contribute to brain repair and cognitive function improvement in patients suffering from traumatic brain injury, $\mathrm{GH}$ deficiency, and neurodegenerative diseases [21-24]. The potential mechanism by which GH/IGF-1 affects the brain is through activating $\mathrm{GH} / \mathrm{IGF}-1$ receptors in the brain, including these on neurons, stem cells, and glial cells, and through the local induction and release of a number of neurotrophic factors, such as vascular endothelial growth factor, epidermal growth factor, and brain-derived neurotrophic factor [25-27]. However, the detailed effects of GH/IGF-1 on brain structure and the relationship between brain structure and serum levels of GH/IGF-1 have not been investigated in humans.

GH-secreting pituitary adenoma (GH-PA) is characterized by excess $\mathrm{GH}$ production and a concomitant increase in IGF-1 levels, leading to enlargement of the extremities, face, and soft tissues, macroglossia and dental changes in patients [28]. The excess circulating GH/IGF-1 levels in patients with GHPA provides a specific model to characterize the effect of excess circulating $\mathrm{GH} / \mathrm{IGF}-1$ on brain gray matter (GM) and white matter (WM) in humans.

In this study, 48 patients with GH-PA, 48 age- and sexmatched clinical non-functional pituitary adenoma (NonFun-PA) patients, and 48 age- and sex-matched healthy controls (HCs) were recruited. We hypothesized that excess circulating GH/IGF-1 levels lead to volume increases in GM and WM, and are significantly related to brain structure volume.

\section{RESULTS}

\section{Demographic and clinical factors}

Forty-eight GH-PA patients, forty-eight age- and sexmatched NonFun-PA patients and forty-eight age- and sex-matched HCs were included. All patients in the GHPA group underwent surgical treatment via the transsphenoidal approach, and had postoperative pathological diagnosis of pituitary GH-secreting adenoma. The demographic and clinical features are shown in Table 1.

\section{The effect of excess GH/IGF-1 production on global brain volume}

Whole-brain analyses provided reliable evidence about whether excess GH/IGF-1 production impacted the entire brain structure, including the TIV, nGMV, nWMV, and nCSFV. As shown in Figure 1A, in our direct comparisons among the three groups, there were no significant differences in TIV. Next, we examined whether the excess serum GH/IGF-1 level differentially affected the three major brain tissue compartments. As shown in Figure 1B-1D, the nGMV and nWMV were significantly greater in the GH-PA group than in the NonFun-PA $(\mathrm{p}<0.01, \mathrm{p}<0.0001)$ and HC groups $(\mathrm{p}<$ $0.0001, \mathrm{p}<0.0001)$. In contrast, the nCSFV in the GHPA group was significantly lower than that in the other groups $(\mathrm{p}<0.0001)$.

\section{The correlation between the three main tissue compartments and excess GH/IGF-1 production}

To detect a relationship between excess GH/IGF-1 production and the three major brain tissue compartments in the GH-PA group, partial correlation analysis was applied. There was a significant positive correlation between GH/IGF-1 level and nGMV (Figure 2A, 2B; $\mathrm{GH}: \mathrm{r}=0.49, \mathrm{p}=0.001$; IGF: $\mathrm{r}=0.5, \mathrm{p}<0.001)$, but a strong negative correlation between GH/IGF-1 level and nCSFV in the GH-PA group (Figure 2E, 2F; GH: $\mathrm{r}=$ $0.43, p=0.003$; IGF: $r=-0.49, p=0.001)$. However, we observed no significant relationship between GH/IGF-1 level and nWMV in the GH-PA group (Figure 2C, 2D; GH: $r=0.17, p=0.24$; IGF: $r=0.28, p=0.054$ ). To detect the relationships between serum GH/IGF-1 levels and the three major brain tissue compartments in adults, partial correlation analysis was performed in the NonFun-PA group. No significant correlations between GH/IGF-1 and nGMV, nWMV, or nCSFV in Figure 2G$2 \mathrm{~L}(\mathrm{p}<0.05)$. Furthermore, we performed partial correlation analysis between brain structures (nGMV, nWMV, nCSFV) and other hypophyseal hormones in the GH-PA group adjusting for age and sex. The results showed no significant relationships between other hypophyseal hormones and brain structures (nGMV, nWMV, nCSFV) in Table 2.

\section{VBM analysis}

\section{GM regional differences}

Other factors such as tumors and natural variations can affect brain structures. Therefore, we first performed 
Table 1. Demographic and clinical characteristics of the participants.

\begin{tabular}{|c|c|c|c|c|}
\hline Characteristic & $\begin{array}{l}\text { GH-PA } \\
(n=48)\end{array}$ & $\begin{array}{c}\text { NonFun-PA } \\
\quad(n=48)\end{array}$ & $\begin{array}{l}\text { Control } \\
(\mathrm{n}=48)\end{array}$ & P-value \\
\hline Age (years) & $43.3(20-68)$ & $43.3(20-68)$ & $43.3(20-68)$ & $1.000^{\mathrm{a}}$ \\
\hline $\operatorname{Sex}(M / F)$ & $24 / 24$ & $24 / 24$ & $24 / 24$ & $1.000^{\mathrm{b}}$ \\
\hline Serum GH (ng/ml) & $14(0.3-40)$ & $0.3(0.1-2.4)$ & NA & $<0.001^{\mathrm{c}}$ \\
\hline Serum IGF-1 (ng/ml) & $748(199-1415)$ & $161(58-437)$ & NA & $<0.001^{\mathrm{c}}$ \\
\hline Serum COR (ng/ml) & $108(40-209)$ & $103(10-228)$ & NA & $0.5489^{\mathrm{c}}$ \\
\hline Serum PRL (ng/ml) & $11(0.5-66.2)$ & $14(0.5-54.5)$ & NA & $0.1698^{\mathrm{c}}$ \\
\hline Serum LH (mIU/ml) & $5.6(0.6-32.6)$ & $6.4(0.1-43.3)$ & NA & $0.6311^{\mathrm{c}}$ \\
\hline Serum FSH (mIU/ml) & $12(0.8-77.9)$ & $16(1.2-145)$ & NA & $0.3831^{\mathrm{c}}$ \\
\hline Serum E2 (pg/ml) & $50(20-176)$ & $50(20-381)$ & NA & $0.9471^{\mathrm{c}}$ \\
\hline Serum P4 (ng/ml) & $0.7(0.2-7.9)$ & $0.4(0.2-4.4)$ & NA & $0.2322^{\mathrm{c}}$ \\
\hline Serum T (ng/ml) & $0.9(0.2-4.2)$ & $1(0.2-5.4)$ & NA & $0.6640^{\mathrm{c}}$ \\
\hline Serum TSH (uTu/ml) & $1.3(0.2-2.9)$ & $1.5(0.2-4.7)$ & NA & $0.2105^{\mathrm{c}}$ \\
\hline Therapy & surgery & NA & NA & NA \\
\hline Pathology & $\mathrm{GH}(+)$ & NA & NA & NA \\
\hline
\end{tabular}

${ }^{a}$ One-way ANOVA; ${ }^{b}$ Fisher's exact test, two-sided; c Student's t-test between GH-PA and NonFun-PA, two-sided. GH, growth hormone; IGF-1, insulin-like growth factor-1; GH-PA, GH-secreting pituitary adenoma; NonFun-PA, non-function pituitary adenoma patients; COR, cortisol; PRL, prolactin; LH, luteinizing hormone; FSH, follicle-stimulating hormone; E2, estradiol; P4, progesterone; T, testosterone; TSH, thyroid stimulating hormone.

A

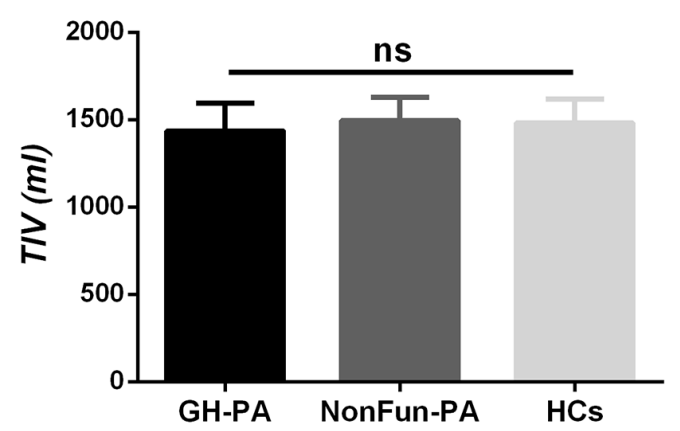

C

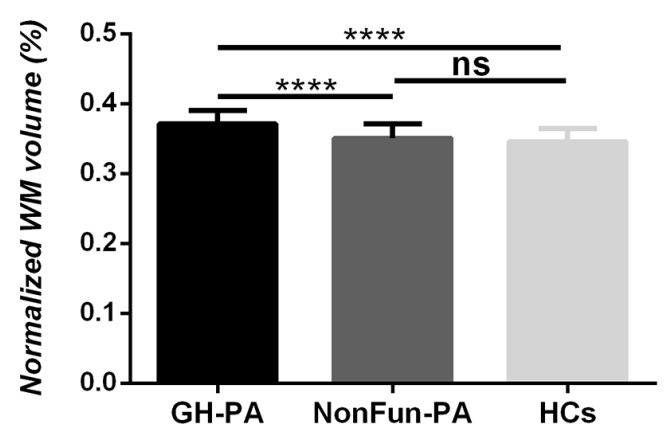

B

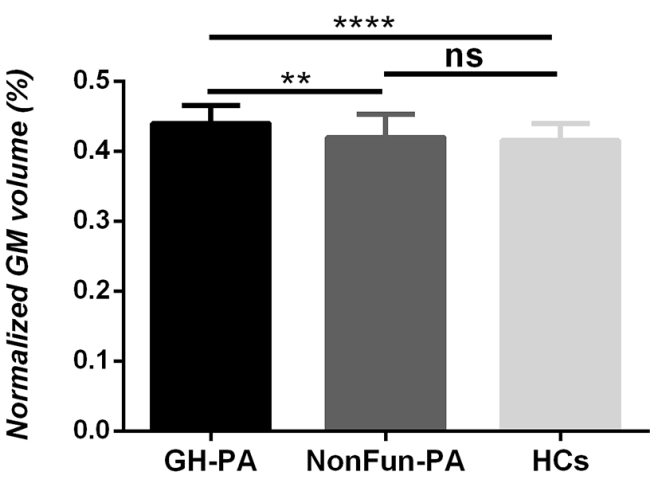

D

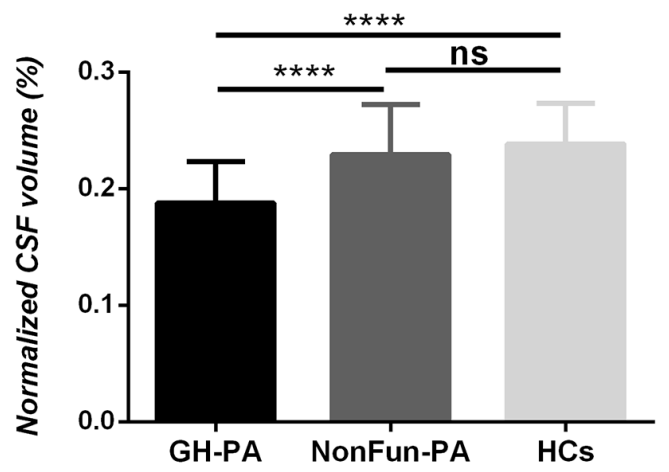

Figure 1. Brain tissue volume changes in patients with GH-PA compared to NonFun-PA and HCs. Statistical analysis of total intracranial volume (TIV) (A), normalized GM volume (nGMV) (B), normalized WM volume (nGWV) (C), and normalized CSF volume (nCSFV) (D) among three groups. Significance was determined by one-way ANOVA with Bonferroni's multiple comparisons test. ns represents no significant difference, $* * \mathrm{p}<0.01, * * * * \mathrm{p}<0.0001$. 
whole-brain VBM analysis at the voxel level through the two-sample t-tests were performed, and found the differences in GMV between GH-PA and HCs groups, the GH-PA and NonFun-PA groups, and the NonFun$\mathrm{PA}$ and HCs groups (Figure 3A-3C). Furthermore, we calculated the similarity of the GMV alteration pattern between the GH-PA and HCs groups and between GHPA and NonFun-PA groups using Pearson's correlation, and found a correlation coefficient of $0.64(p<0.0001$, Figure 3D). VBM analysis showed extensive bilateral
A

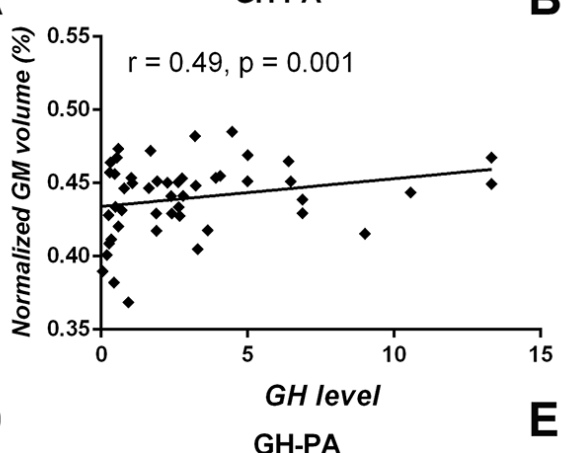

D

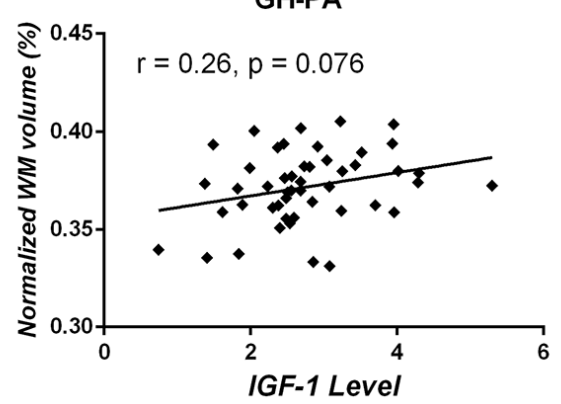

G

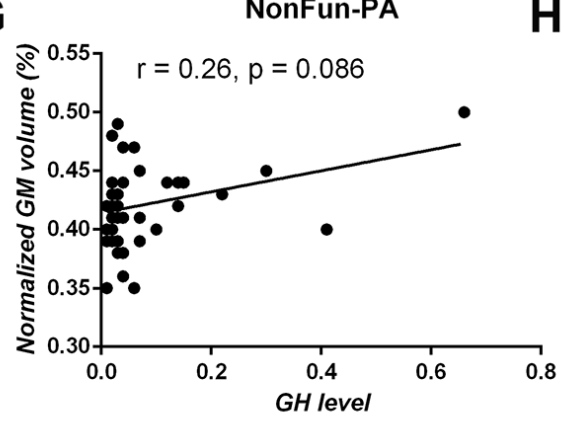

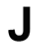

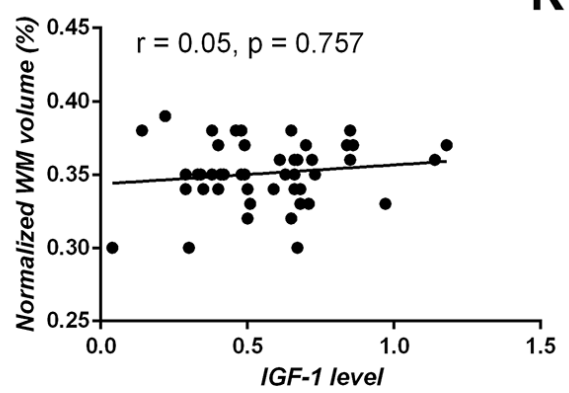

B

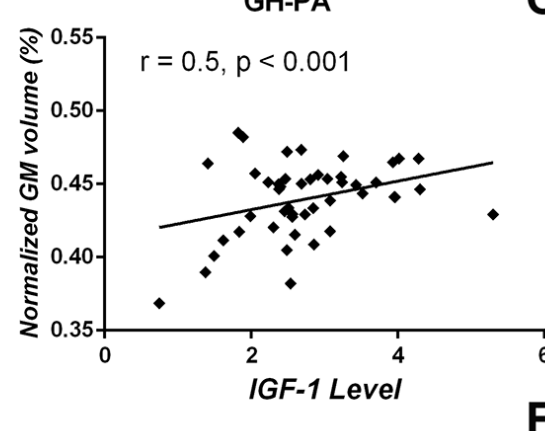

GH-PA

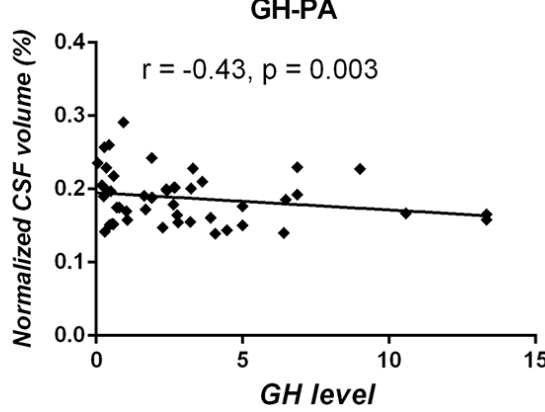

NonFun-PA

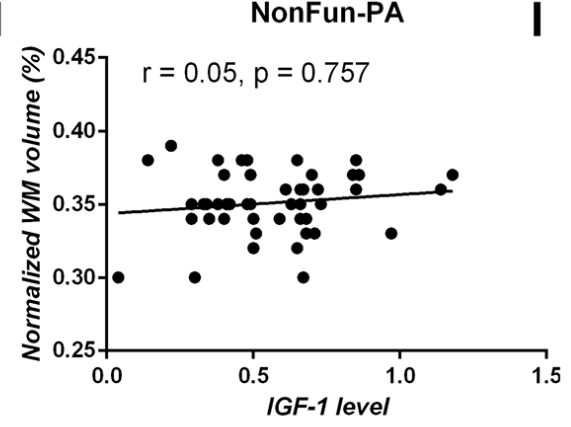

NonFun-PA

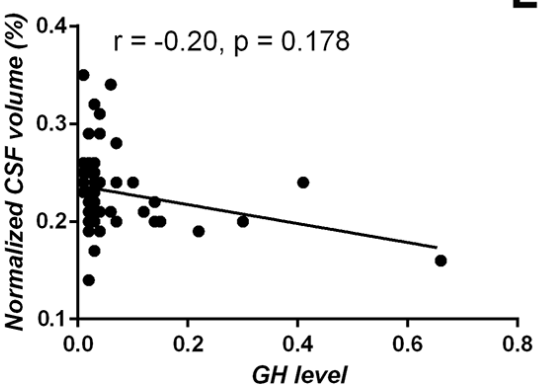

F

L
C
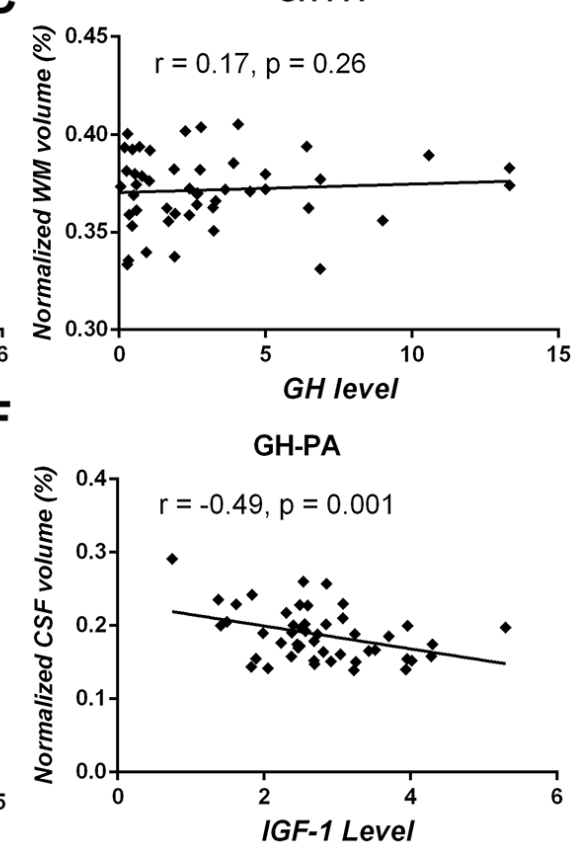

NonFun-PA
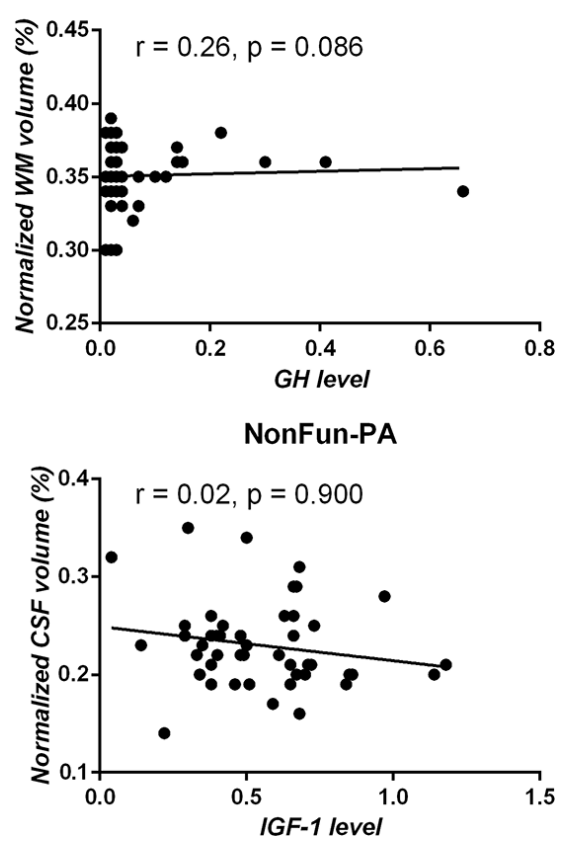

Figure 2. Correlation analysis between serum GH/IGF-1 levels and brain tissue volume in patients with GH-PA and patients with Nonfun-PA groups. The normalized GM volume (nGMV) shows significant positive correlation with GH (A) and IGF-1 (B) in patients with GH-PA. The normalized WM volume (nGWV) shows no significant correlation with GH (C) or IGF-1 (D) in patients with GH-PA. The normalized CSF volume (nCSFV) shows significant negative correlation with GH (E) and IGF-1 (F) in patients with GH-PA. In patients with NonFun-PA, nGMV, nWMV, and nCSFV show no significant correlation with GH/IGF-1 (G-L). 
Table 2. Partial correlation analysis between hypophyseal hormones and brain structure (nGMV, nWMV, nCSFV) in GH-PA patients adjusting for age and sex.

\begin{tabular}{|c|c|c|c|c|c|c|}
\hline \multirow{2}{*}{ Correlation } & \multicolumn{2}{|c|}{ nGMV } & \multicolumn{2}{|c|}{ nWMV } & \multicolumn{2}{|c|}{ nCSF } \\
\hline & r-value & p-value & r-value & p-value & r-value & p-value \\
\hline Serum COR (ng/ml) & -0.264 & 0.076 & 0.006 & 0.967 & 0.176 & 0.241 \\
\hline Serum PRL (ng/ml) & 0.040 & 0.794 & 0.052 & 0.732 & -0.056 & 0.712 \\
\hline Serum LH (mIU/ml) & 0.054 & 0.720 & 0.039 & 0.798 & -0.053 & 0.724 \\
\hline Serum FSH (mIU/ml) & 0.068 & 0.652 & 0.092 & 0.544 & -0.095 & 0.529 \\
\hline Serum E2 (pg/ml) & 0.199 & 0.184 & 0.022 & 0.887 & -0.144 & 0.340 \\
\hline Serum P4 (ng/ml) & 0.023 & 0.880 & 0.186 & 0.217 & -0.120 & 0.425 \\
\hline Serum T (ng/ml) & -0.252 & 0.091 & -0.189 & 0.207 & 0.282 & 0.058 \\
\hline Serum TSH (uTu/ml) & -0.073 & 0.630 & -0.118 & 0.435 & 0.121 & 0.422 \\
\hline
\end{tabular}

COR, cortisol; PRL, prolactin; LH, luteinizing hormone; FSH, follicle-stimulating hormone; E2, estradiol; P4, progesterone; T, testosterone; TSH, thyroid stimulating hormone.

clusters of significantly increased GMV along the midline regions from the frontal cortex to parietal cortex, subcortical GM, and cerebellum GM in the GHPA group compared with those in the other two groups (FDR-corrected, $\mathrm{p}<0.05$, Supplementary Figure 1A, 1B). No significant GMV alterations were observed in the cortex between NonFun-PA and HCs groups, except for in the regions close to the tumor. No cluster was identified to have a significantly lower GMV in the GHPA group than in the controls.

\section{WM regional differences}

Similarly, Figure 4A-4C showed WMV alterations between the GH-PA and HCs groups, the GH-PA and NonFun-PA groups, and the NonFun-PA and HCs groups, respectively, using whole-brain VBM analysis at the voxel level. The WMV alteration pattern between the GH-PA and HCs groups and between the GH-PA and NonFun-PA groups showed a correlation coefficient of 0.69 ( $p<0.0001$, Figure 4D). VBM analysis showed revealed extensive bilateral clusters of significantly
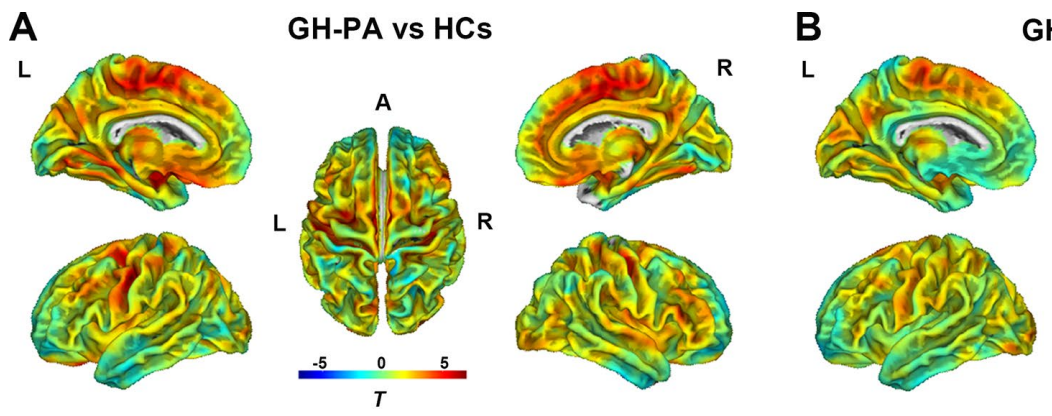

GH-PA vs NonFun-PA
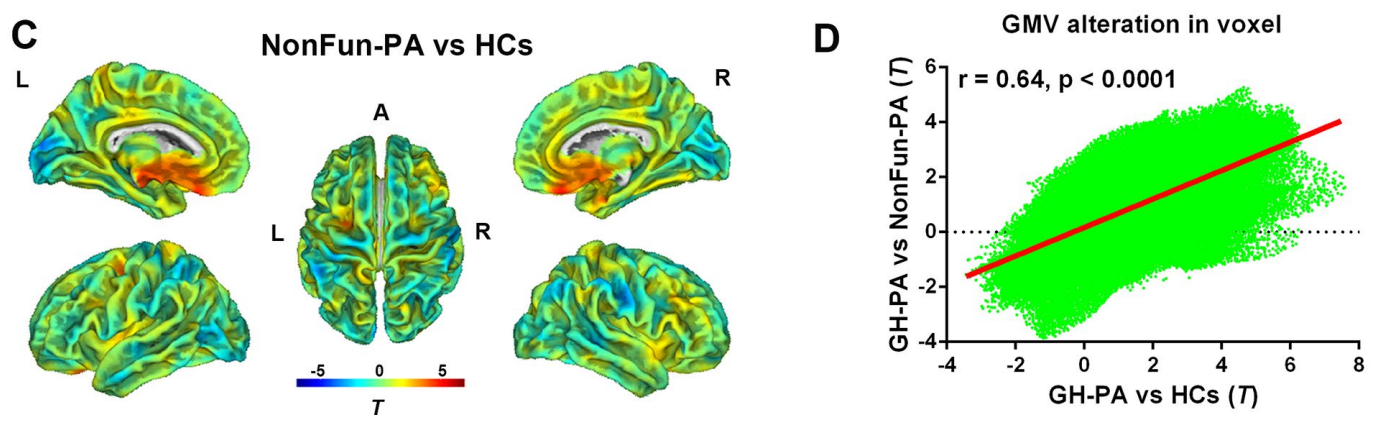

Figure 3. Distribution of GMV alterations in voxel level among the three groups. VBM analysis showing the GMV alteration in GHPA vs HCs (A), in GH-PA vs NonFun-PA (B), in NonFun-PA vs HCs (C). Correlation analysis of GMV alteration pattern between GH-PA vs HCs and GH-PA vs NonFun-PA (D). 
increased WMV in the entire brain, including the cerebellum and brain stem, in GH-PA compared to other two groups (Supplementary Figure 2A, 2B, FDR corrected, $\mathrm{p}<0.05)$. There were no clusters with significant differences in WMV between the NonFun-PA and $\mathrm{HC}$ groups.

\section{RBM analysis}

Next, we studied the detailed effects of excess serum GH/IGF-1 levels on the GMV and WMV in different brain regions by comparing the patients with GH-PA and HCs. RBM analysis was used to obtain the average volume of each brain region, according to Hammers' atlas maps (a total of 68 brain regions). As shown in Figure 5A, the GMV of 54 brain regions (Supplementary Table 1) was significantly greater in the patients than in the HCs. As shown in Figure 5B, as assessment of the $\mathrm{WM}$ revealed that the WMV of 54 brain regions (Supplementary Table 1) was significantly greater in the patients than in the HCs.

\section{Relation of average GMV/WMV in the brain regions with excess GH/IGF-1 production}

Furthermore, we performed correlation analyses between GH/IGF-1 levels and brain region volume based on
A

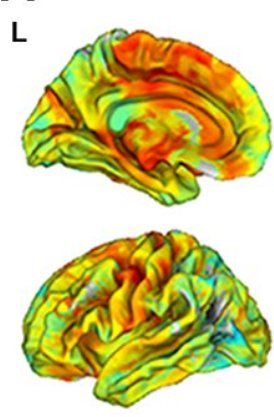

GH-PA vs HCs

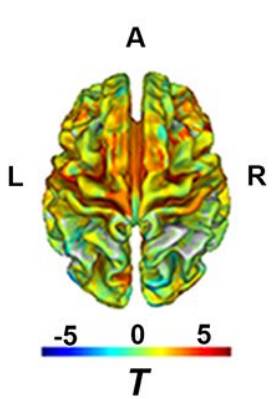

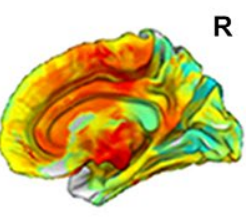

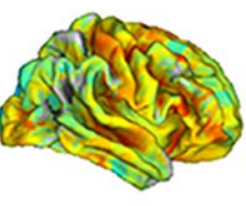

C

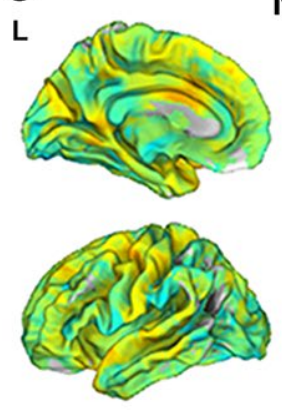

NonFun-PA vs HCs

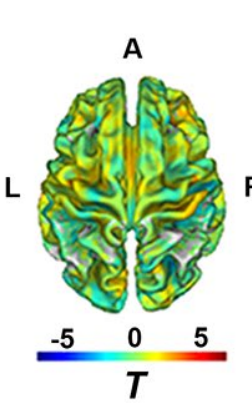

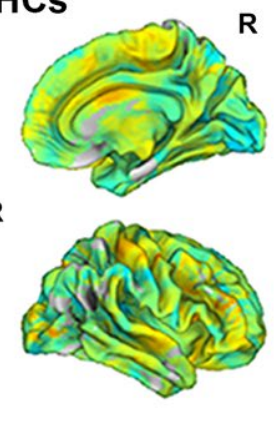

the results of the RBM analysis. As shown in Supplementary Figure 3, the GMV of 18 brain regions (Supplementary Table 2) were significantly positively correlated with serum GH levels $(\mathrm{p}<0.05)$. Meanwhile, the GMV of 19 brain regions (Supplementary Table 2) were significantly positively correlated with serum IGF1 levels ( $\mathrm{p}<0.05$ ). Regarding WM, as shown in Supplementary Figure 4, there were only 5 brain regions (Supplementary Table 3 ) was positively correlated with GH levels $(\mathrm{p}<0.05)$, and 10 brain regions (Supplementary Table 3 ) was positively correlated with IGF-1 levels $(\mathrm{p}<0.05)$.

\section{DISCUSSION}

In the present study, the results showed the following: (a) Whole GMV and WMV are significantly increased at the expense of CSFV in patients with excess GH/IGF-1 production; (b) the increase in GMV and the reduction in CSFV were significantly correlated with serum GH/IGF1 levels; (c) VBM showed that excess serum GH/IGF-1 levels can lead to widespread increases in GMV and WMV in patients with GH-PA; (d) at the regional level, the GMV and WMV of 54 brain regions (from a total of 68 brain regions) exhibited obvious increases due to excess serum GH/IGF-1 levels; (e) of all of the brain regions examined, the GMV in only a few of these brain
B

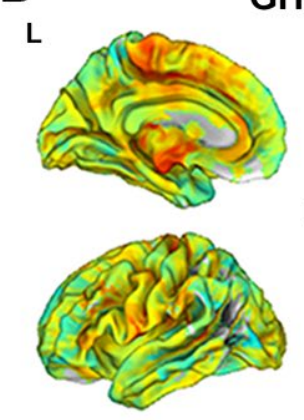

GH-PA vs NonFun-PA
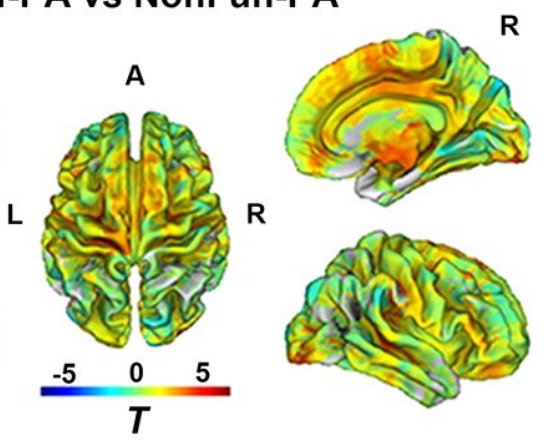

D

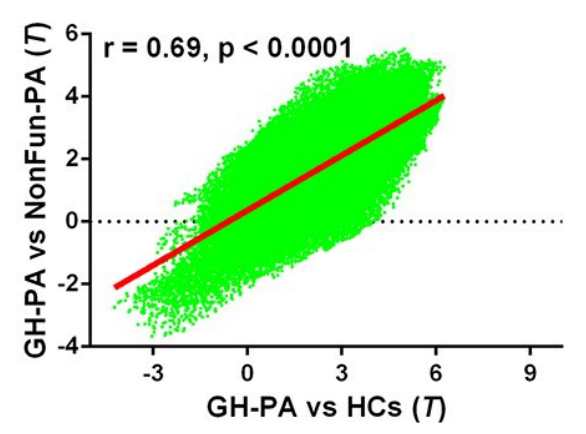

Figure 4. Distribution of WMV alteration in voxel level among three groups. VBM analysis showing the WMV alteration in GH-PA vs HCs (A), in GH-PA vs NonFun-PA (B), in NonFun-PA vs HCs (C). Correlation analysis of WMV alteration between GH-PA vs HCs and GH-PA vs NonFun-PA (D). 


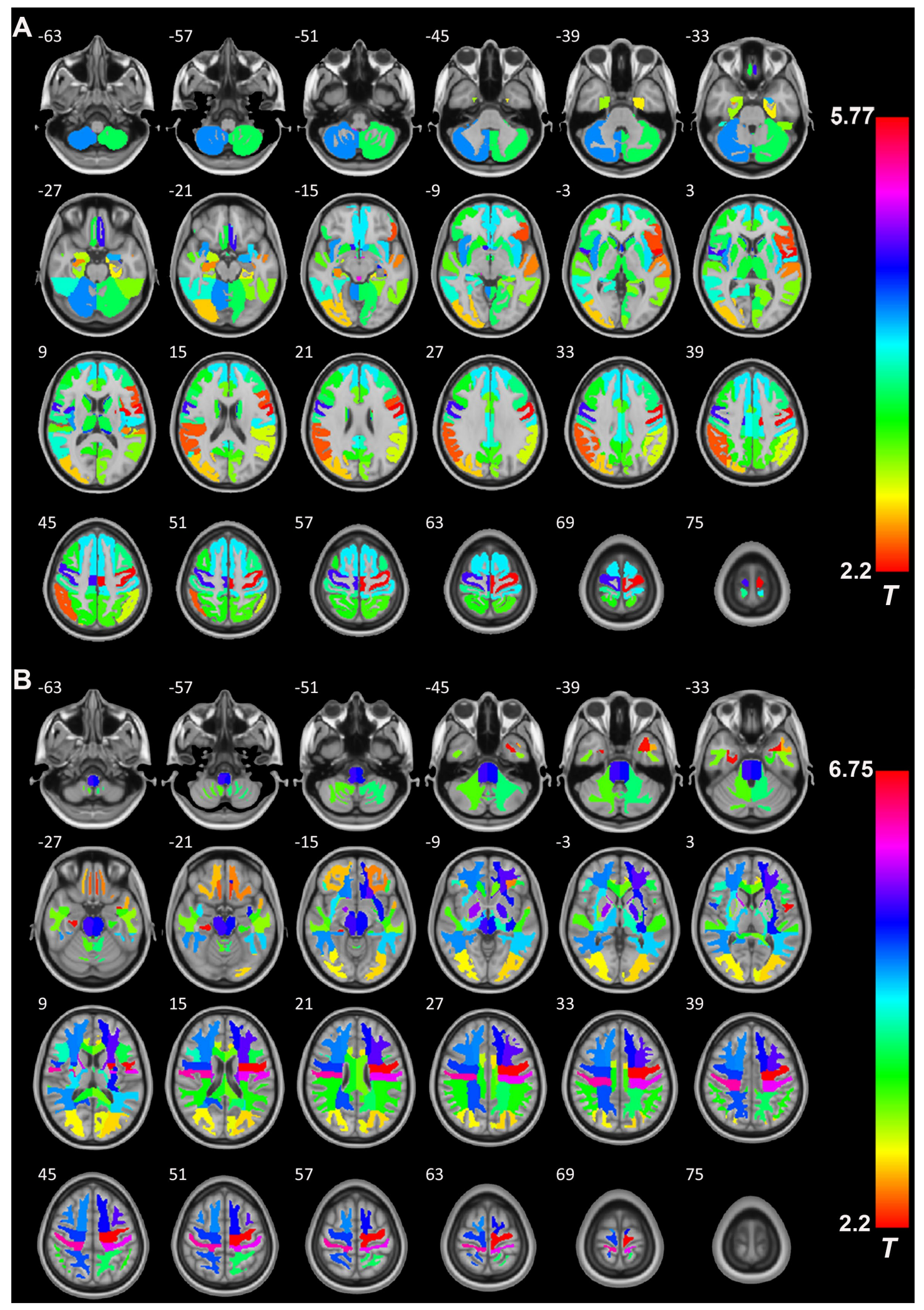

Figure 5. The role of GH/IGF-1 in the GMV and WMV of brain regions. (A) RBM analysis showing the increased GMV of 54 brain regions (Supplementary Table 1, from a total of 68 brain regions, Hammers' atlas) in patients with excess GH/IGF-1 production compared to that in HCs. (B) RBM analysis showing the white matter volume (WMV) of 54 brain regions (Supplementary Table 1, from a total of 68 brain regions, Hammers' atlas) increase in patients with excess GH/IGF-1 production compared to HCs. Significance was determined by uncorrected $p<0.05$, using two-sample t-tests. 
regions showed striking correlations with $\mathrm{GH} / \mathrm{IGF}-1$ levels (GH, 18 brain regions; IGF-1, 19 brain regions). The findings from this study provide imaging evidence that GH and IGF-1 contribute to brain tissue growth in adult humans.

In our study, whole-brain analyses showed no TIV increase in patients with excess GH/IGF-1 levels, but significant increases in GMV and WMV at the expense of CSFV, as well as significant correlations between GH/IGF-1 and GMV/CSFV. The lack of a difference in TIV may have been because the groups were composed of age- and sex-match adults (20-68 years old) subjects, and the intracranial volume has been shown to be fixed since adolescence [30, 31]. Considering the potential effects of others hypophyseal hormones on human brain structures, partial correlation analysis was performed between brain structure and others hypophyseal hormones adjusting for age and sex $[32,33]$. Our results showed no significant relationships between others hypophyseal hormone and brain structures, further demonstrating the definite effects of higher GH/IGF-1 levels on brain structure. The increase in brain tissue (GM, WM) in patients with excess GH/IGF-1 production may be attributed to the roles of GH/IGF-1 in stimulating protein synthesis and proliferation in neurons, glia, and oligodendrocytes, favoring neuronal survival, and inhibiting apoptosis as reported in animal studies and GH/IGF-1 are still vital regulators of brain structure in the postadolescence period [34]. In the patients with NonFun-PA, the lack of a significant relationship between $\mathrm{GH} / \mathrm{IGF}-1$ and brain structure may be due to the lower serum GH/IGF-1 level, and reduction of GH/IGF-1 receptor in the brain regions with increasing age. In humans and animals, aging is characterized by a progressive decrease in both GH/IGFI levels and brain tissue (GM and white WM) volume and an increase in CSFV $[11,35,36]$. In our study, correlation analyses indicated that higher GH/IGF-1 levels were accompanied by a greater GMV and lower CSFV in GH-PA patients. These results suggest that maintaining the higher serum level of GH/IGF-1 may alleviate brain atrophy in aged humans.

Considering the effects of other factors, such as tumors and population characteristics, on brain structure, we calculated the similarity of the GMV alteration in voxels between GH-PA vs HCs and GH-PA vs NonFun-PA, and the results showed significant positive correlation. This finding indicates the universal effect of higher GH/IGF-1 levels on brain grey matter of population in voxel level. In our global GM analysis with corrected, VBM showed extensive bilateral clusters of significantly increased GMV along the midline regions from the frontal cortex to parietal cortex, subcortical GM, and cerebellum GM in patients with excess GH/IGF-1 production. The increase in volume in the extensive number of regions may be due to the expression of GH/IGF-1 receptors are modulated by higher GH/IGF-1 level [37]. The animal studies have reported that $\mathrm{GH} / \mathrm{IGF}-1$ receptors are throughout the entire brain, and mainly concentrated in the superficial and deep cortical layers, cerebellum, olfactory bulb, amygdala, thalamic nuclei, brain stem and hippocampus $[4,5]$. We further study the effect of GH/IGF-1 on specific brain regions (Hammers' atlas) with RBM analysis, the results showed that 54 brain regions from a total of 68 brain regions increased the GMV. These findings are generally in line with those of other studies which reported GH or IGF-1 gene knock-out mice showed certain areas of the brain are significantly smaller compared to wild-type controls $[13,14]$. The non-affected regions (only 14 in total) included the bilateral anterior medial temporal lobe, anterior lateral temporal lobe, inferior middle temporal gyrus, fusiform gyrus, pallidum, corpus callosum, and orbitofrontal gyrus. One previous study have reported that distribution of lowest IGF-1 receptor density in pallidum and corpus callosum compared to others regions being studied [38]. We speculate that no significant increase of these regions volume may be due to lower distribution of $\mathrm{GH} / \mathrm{IGF}-1$ receptor. Taken together, our results provide clear evidence that higher GH/IGF-1 levels can lead to increase of grey matter volume in various brain regions in adult humans.

In neurodegenerative diseases, such as amyotrophic lateral sclerosis (ALS) and Alzheimer's disease (AD), the role of GH/IGF-1 has also been reported. In animal studies, some researchers have suggested that GH/IGF-1 administration can decrease Ab1-40 protein levels, which may contribute to age-related cognitive deficits, and promote improvements in learning and memory in aged animals with AD [39]. Similarly, a few clinical studies have reported that a dramatic decline in serum GH/IGF-1 levels in patients with $\mathrm{AD}$ and subsequent IGF-1 administration could provide significant benefits to these patients $[24,40]$. In patients affected by ALS, a significant reduction in $\mathrm{GH} / \mathrm{IGF}-1$ secretion was found, and the efficacy of GH/IGF-1 treatment in improving muscle force, motor coordination, and protection of motor neurons has been controversial in the clinic [41, 42]. Meanwhile, structural MRI analyses have shown multiple brain regions with decreased GMV, and there was a significant correlation between brain atrophy and neurological dysfunction in conditions such as ALS, AD, Parkinson's disease, and prion disorders. For example, restricted regional GM atrophy mainly in the medial temporal structures, including the bilateral hippocampus, parahippocampal gyrus, amygdala and entorhinal cortex, as well as the posterior cingulate gyrus and medial thalamus, and reductions in WMV in the left parahippocampal gyrus extending to the temporal WM, 
right temporal $\mathrm{WM}$ extending to the parahippocampal gyrus and posterior corpus callosum, were found in patients with AD by VBM analysis [43, 44]. In patients with ALS, VBM analysis showed that diffuse GMV reduction in primary motor cortex bilaterally, frontotemporal areas, cerebellum and basal ganglia [45]. Interestingly, our results indicated that higher serum GH/IGF-1 level can markedly enhance the volume of these brain regions, such as the bilateral hippocampus, amygdalae, entorhinal cortex, thalamus, precentral gyrus, insula, and posterior cingulate gyrus, using structural MRI analysis in humans. Furthermore, the correlation test showed a significant positive association between GH/IGF-1 with some of these brain regions volume. These findings suggest that GH/IGF-1 should be considered as a potential preventive and therapeutic strategy for various neurodegenerative diseases, but its clinical application require further study.

GH/IGF-1 was shown to contribute to the proliferation of oligodendrocytes, axonal regeneration, and myelination in experimental studies [46-48]. In humans, diffusion tensor imaging (DTI) analysis showed WM recovery after traumatic brain injury (TBI) was greater in patients with higher serum IGF-I levels [49]. In our study, we observed there were significant correlation of WMV alteration between GH-PA vs HCs and GH-PA vs NonFun-PA, as well as significantly increased volumes in widespread brain WM regions in the patients with high levels of GH/IGF-1 using VBM and RBM analysis. However, the correlation analysis showed no significant relationship between nWMV and excess circulating GH/IGF-1 levels. The role of excess circulating GH/IGF1 levels in axon and myelination need further study due to the WMV increase can't reflect the detail alteration of axon and myelination.

In present study, there are some limitations. Firstly, lack of diffusion tensor imaging (DTI) data to analysis of the effect of excess circulating GH/IGF-1 levels on axonal structural integrity. Second, no longitudinal imaging data collected from patients with GH-secreting pituitary adenoma after surgery to study whether the decrease of serum level of GH/IGF-1 lead to the alteration of brain structure. Third, there are no neuropsychological and cognitive assessment in the subjects to investigate the effect of higher GH/IGF-1 on cognitive function.

\section{CONCLUSIONS}

High serum GH/IGF-1 levels significantly increased GMV and WMV in most regions and strikingly correlated with brain structure in adult humans. Our results provide imaging evidence that serum $\mathrm{GH} / \mathrm{IGF}-1$ contributes to brain growth, indicating that GH/IGF-1 may be a potential treatment option for neurodegenerative disorders and brain injury in humans.

\section{MATERIALS AND METHODS}

\section{Participants}

Forty-eight patients with GH-PA, who accepted therapy in the department of neurosurgery, Beijing Tiantan Hospital, were enrolled. The inclusion criteria for the patient group were being 18-70 years of age and having a diagnosis of GH-PA, according to clinical manifestations, GH/IGF-1 serum levels, and postoperative pathological diagnosis. Forty-eight age- and sex-matched clinical NonFun-PA patients and forty-eight age- and sexmatched healthy controls (HCs) constituted the control groups. The exclusion criteria for the patient and control groups included a history of stroke, cerebral trauma, or other intracranial space-occupying lesions; neurodegenerative diseases; substance use disorders for alcohol or heroin; and an inability to complete the MRI examinations.

The Beijing Tiantan hospital ethics committee approval and informed written consent from the participants were obtained, in accordance with the Declaration of Helsinki. The relevant guidelines and regulations were strictly observed in our experimental procedures.

\section{Endocrine evaluation}

All patients had completed endocrine testing (GH, IGF-1, cortisol, prolactin, luteinizing hormone, follicle-stimulating hormone, estradiol, progesterone, testosterone, thyroid stimulating hormone) for the study. Venous blood samples were collected between 06:00 a.m. and 10:00 a.m. following 10-12 $\mathrm{h}$ of fasting. The serum value of hormones were measured using the IMMULITE 2000 immunoassay system (Siemens). To remove the effect of age and sex, IGF-1 and GH levels were calculated as follows: serum IGF-1 or GH value/95th percentile of the age- and sex-adjusted normal range [29].

\section{Image acquisition}

All patients were scanned on the 3T Philips Ingenia CX MRI scanner (Philips Healthcare, Best, the Netherlands) using a commercial 32-channel head coil. The T1weighted (T1W) images were obtained with a 3D sagittal magnetization-prepared rapid acquisition gradient echo (MPRAGE) sequence (resolution of $1 \mathrm{~mm}$ isotropic voxels; field of view $=240 \times 240 \mathrm{~mm}^{2} ; 196$ slices; flip angle $=8^{\circ}$; inversion time $(\mathrm{TI}) /$ repetition time $(\mathrm{TR}) /$ echo time $(\mathrm{TE})=494 / 1339 / 3.0 \mathrm{~ms}$; data matrix $=$ $240 \times 240$; bandwidth $=241 \mathrm{~Hz} / \mathrm{px} ;$ SENSE = 2). The 
structural images of all participants were examined for possible lesions in the cortex, as an exclusion criterion, by two expert radiologists.

\section{Data preprocessing and analysis}

Image data processing was conducted using SPM12 (http://www.fil.ion.ucl.ac.uk/spm) and the CAT12 toolbox (http://www.neuro.uni-jena.de/cat/) in the MATLAB environment (MATHWORKS, California, USA). First, using the module "Segment Data" of CAT12, we segmented every T1W image into GM, WM, and CSF. The modulated warped GM images were then normalized to Montreal Neurological Institute (MNI)-152 standard space with an isotropic voxel resolution of $1.5 \mathrm{~mm} \times 1.5 \mathrm{~mm} \times 1.5 \mathrm{~mm}$. Next, using "Display one slice for all images", we checked the data quality to determine if some reasonable results were obtained after the segmentation and normalization procedures. Using a boxplot and correlation matrices, we also checked sample homogeneity to identify outliers by visualizing the correlation between the volumes. The modulated GM map of each individual was smoothed with an 8-mm full-width at half-maximum Gaussian kernel.

The absolute GM volume (GMV), absolute WM volume (WMV), and absolute CSF volume (CSFV) as well as total intracranial volume (TIV) of each subject were estimated using the "Estimate TIV" module. To correct for variation in subjects' head sizes, we calculated the normalized GM volume (nGMV), normalized WM volume (nWMV), and normalized CSF volume (nCSFV) by dividing the individual subject's GMV, WMV, and CSFV values by each subject's respective TIV value. TIV, nGMV, nWMV, and nCSFV were compared among the three groups using one-way ANOVA with Bonferroni's multiple comparisons test. The statistical analyses were performed using Statistical Package for Social Sciences software (SPSS, ver. 25.0; Chicago, IL) with $\mathrm{p}<0.05$ as the significance level.

\section{Voxel-based morphometry (VBM) analysis}

A two-sample t-test was applied in CAT12 to assess significant differences among the three groups with TIV, age, and sex as covariates. We calculated the similarity of the GMV/WMV alteration pattern between GH-PA and HCs and between GH-PA and NonFun-PA using Pearson's correlation based on $T$ values of the group level analysis in the whole brain. To further confirm the difference in GMV/WMV between two groups, significance was determined using positive false discovery rate $(\mathrm{pFDR})<0.05$ at the voxel level and cluster sizes $\mathrm{k}>333$.

\section{Region-based morphometry (RBM) analysis}

The averaged GMV, WMV and CSFV from each region were extracted according to the Hammers atlas (threedimensional maximum probability atlas of the human brain, with particular reference to the temporal lobe; Hammers, 2003). The normalized volume of each brain region between patients and controls was compared using two-sample t-tests. The statistical analyses were performed using SPSS with $\mathrm{p}<0.05$ as the significance level.

\section{Correlation analysis}

Partial correlation analysis was performed to determine the correlation between the three main tissue compartments (nGMV, nWMV, nCSFV) and serum hormone levels (GH, IGF-1, cortisol, prolactin, luteinizing hormone, follicle-stimulating hormone, estradiol, progesterone, testosterone, thyroid stimulating hormone) in patients with GH-PA and NonFun-PA adjusting for age and sex. $\mathrm{p}<0.05$ was considered statistically significant, and analyses were conducted using SPSS.

\section{Abbreviations}

GH: growth hormone; IGF-1: insulin-like growth factor-1; GH-PA: GH-secreting pituitary adenoma; NonFun-PA: non-functional pituitary adenoma; VBM: voxel-based morphometry; RBM: region-based morphometry; nGMV: normalized gray matter volume; nWMV: normalized white matter volume; nCSFV: normalized cerebrospinal fluid volume; TIV: total intracranial volume.

\section{ACKNOWLEDGMENTS}

We thank the Department of Neuropathology, Beijing Neurosurgical Institute, Beijing Tiantan Hospital for providing pathological information.

\section{CONFLICTS OF INTEREST}

All the authors declare that they have no conflicts of interest.

\section{FUNDING}

This study was supported by the Ministry of Science and Technology of China (Grant 2015CB351701), National Nature Science Foundation of China (Grant 91132302), Beijing Municipal Science and Technology Commission (Grant Z171100000117002), and National Natural Science Foundation of China (Grant 81601205). 


\section{REFERENCES}

1. Giustina A, Veldhuis JD. Pathophysiology of the neuroregulation of growth hormone secretion in experimental animals and the human. Endocr Rev. 1998; 19:717-97.

https://doi.org/10.1210/er.19.6.717

PMID: $\underline{961545}$

2. Sonntag WE, Ramsey M, Carter CS. Growth hormone and insulin-like growth factor-1 (IGF-1) and their influence on cognitive aging. Ageing Res Rev. 2005; 4:195-212.

https://doi.org/10.1016/i.arr.2005.02.001

PMID: 16024298

3. Ashpole NM, Sanders JE, Hodges EL, Yan H, Sonntag WE. Growth hormone, insulin-like growth factor-1 and the aging brain. Exp Gerontol. 2015; 68:76-81. https://doi.org/10.1016/i.exger.2014.10.002 PMID:25300732

4. Bondy C, Werner H, Roberts CT Jr, LeRoith D. Cellular pattern of type-I insulin-like growth factor receptor gene expression during maturation of the rat brain: comparison with insulin-like growth factors I and II. Neuroscience. 1992; 46:909-23.

https://doi.org/10.1016/0306-4522(92)90193-6 PMID: 1311816

5. Lobie PE, García-Aragón J, Lincoln DT, Barnard R, Wilcox JN, Waters MJ. Localization and ontogeny of growth hormone receptor gene expression in the central nervous system. Brain Res Dev Brain Res. 1993; 74:225-33.

https://doi.org/10.1016/0165-3806(93)90008-X PMID:403384

6. Pan W, Yu Y, Cain CM, Nyberg F, Couraud PO, Kastin AJ. Permeation of growth hormone across the blood-brain barrier. Endocrinology. 2005; 146:4898-904.

https://doi.org/10.1210/en.2005-0587 PMID:16099858

7. Nagaraja TN, Patel P, Gorski M, Gorevic PD, Patlak CS, Fenstermacher JD. In normal rat, intraventricularly administered insulin-like growth factor-1 is rapidly cleared from CSF with limited distribution into brain. Cerebrospinal Fluid Res. 2005; 2:5.

https://doi.org/10.1186/1743-8454-2-5

PMID:16045806

8. Armstrong CS, Wuarin L, Ishii DN. Uptake of circulating insulin-like growth factor-I into the cerebrospinal fluid of normal and diabetic rats and normalization of IGF-II mRNA content in diabetic rat brain. J Neurosci Res. 2000; 59:649-60.

https://doi.org/10.1002/(sici)1097-

4547(20000301)59:5<649::aid-jnr8>3.0.co;2-w

PMID:10686593
9. Nylander E, Zelleroth S, Nyberg F, Grönbladh A, Hallberg M. The Protective and Restorative Effects of Growth Hormone and Insulin-Like Growth Factor-1 on Methadone-Induced Toxicity In Vitro. Int J Mol Sci. 2018; 19:E3627.

https://doi.org/10.3390/ijms19113627

PMID:30453639

10. Nyberg F, Hallberg M. Growth hormone and cognitive function. Nat Rev Endocrinol. 2013; 9:357-65.

https://doi.org/10.1038/nrendo.2013.78

PMID:23629538

11. Gasperi M, Castellano AE. Growth hormone/insulin-like growth factor I axis in neurodegenerative diseases. J Endocrinol Invest. 2010; 33:587-91.

https://doi.org/10.1007/bf03346653 PMID:20930497

12. Aberg ND, Brywe KG, Isgaard J. Aspects of growth hormone and insulin-like growth factor-I related to neuroprotection, regeneration, and functional plasticity in the adult brain. ScientificWorldJournal. 2006; 6:53-80.

https://doi.org/10.1100/tsw.2006.22 PMID:16432628

13. Beck KD, Powell-Braxton L, Widmer HR, Valverde J, Hefti F. Igf1 gene disruption results in reduced brain size, CNS hypomyelination, and loss of hippocampal granule and striatal parvalbumin-containing neurons. Neuron. 1995; 14:717-30.

https://doi.org/10.1016/0896-6273(95)90216-3 PMID:

14. Ransome MI, Goldshmit Y, Bartlett PF, Waters MJ, Turnley AM. Comparative analysis of CNS populations in knockout mice with altered growth hormone responsiveness. Eur J Neurosci. 2004; 19:2069-79. https://doi.org/10.1111/i.0953-816x.2004.03308.x PMID:15090034

15. Le Grevès $M$, Zhou $Q$, Berg $M$, Le Grevès $P$, Fhölenhag K, Meyerson B, Nyberg F. Growth hormone replacement in hypophysectomized rats affects spatial performance and hippocampal levels of NMDA receptor subunit and PSD-95 gene transcript levels. Exp Brain Res. 2006; 173:267-73. https://doi.org/10.1007/s00221-006-0438-2 PMID:16633806

16. Grönbladh A, Johansson J, Nöstl A, Nyberg F, Hallberg $\mathrm{M}$. GH improves spatial memory and reverses certain anabolic androgenic steroid-induced effects in intact rats. J Endocrinol. 2013; 216:31-41.

https://doi.org/10.1530/JOE-12-0315

PMID:23092877

17. Bluthé RM, Frenois $F$, Kelley $K W$, Dantzer $R$. Pentoxifylline and insulin-like growth factor-I (IGF-I) abrogate kainic acid-induced cognitive impairment in mice. J Neuroimmunol. 2005; 169:50-8. 
https://doi.org/10.1016/i.jneuroim.2005.07.017 PMID:16154639

18. Malek M, Zahedi Asl S, Sarkaki A, Farbood Y, Doulah AH. The effect of intra-hippocampal injection of growth hormone on spatial learning and memory in animal model of Alzheimer's disease. Pak J Biol Sci. 2009; 12:1237-45.

https://doi.org/10.3923/pjbs.2009.1237.1245

PMID:20384276

19. Heredia M, Fuente A, Criado J, Yajeya J, Devesa J, Riolobos AS. Early growth hormone (GH) treatment promotes relevant motor functional improvement after severe frontal cortex lesion in adult rats. Behav Brain Res. 2013; 247:48-58.

https://doi.org/10.1016/i.bbr.2013.03.012

PMID:23518437

20. Isgaard J, Aberg D, Nilsson M. Protective and regenerative effects of the GH/IGF-I axis on the brain. Minerva Endocrinol. 2007; 32:103-13.

PMID: $\underline{17557036}$

21. High WM Jr, Briones-Galang M, Clark JA, Gilkison C, Mossberg KA, Zgaljardic DJ, Masel BE, Urban RJ. Effect of growth hormone replacement therapy on cognition after traumatic brain injury. J Neurotrauma. 2010; 27:1565-75.

https://doi.org/10.1089/neu.2009.1253 PMID:20578825

22. Scheepens A, Williams CE, Breier BH, Guan J, Gluckman PD. A role for the somatotropic axis in neural development, injury and disease. J Pediatr Endocrinol Metab. 2000; 13:1483-91.

https://doi.org/10.1515/ipem-2000-s623

PMID: 11202225

23. Falleti MG, Maruff $P$, Burman $P$, Harris $A$. The effects of growth hormone $(\mathrm{GH})$ deficiency and $\mathrm{GH}$ replacement on cognitive performance in adults: a meta-analysis of the current literature. Psychoneuroendocrinology. 2006; 31:681-91.

https://doi.org/10.1016/j.psyneuen.2006.01.005 PMID:16621325

24. McGinley LM, Sims E, Lunn JS, Kashlan ON, Chen KS, Bruno ES, Pacut CM, Hazel T, Johe K, Sakowski SA, Feldman EL. Human Cortical Neural Stem Cells Expressing Insulin-Like Growth Factor-I: A Novel Cellular Therapy for Alzheimer's Disease. Stem Cells Transl Med. 2016; 5:379-91.

https://doi.org/10.5966/sctm.2015-0103

PMID:26744412

25. Devesa J, Almengló C, Devesa P. Multiple Effects of Growth Hormone in the Body: Is it Really the Hormone for Growth? Clin Med Insights Endocrinol Diabetes. 2016; 9:47-71.
https://doi.org/10.4137/CMED.S38201 PMID:27773998

26. Lichtenwalner RJ, Forbes ME, Sonntag WE, Riddle DR. Adult-onset deficiency in growth hormone and insulinlike growth factor-I decreases survival of dentate granule neurons: insights into the regulation of adult hippocampal neurogenesis. J Neurosci Res. 2006; 83:199-210.

https://doi.org/10.1002/inr.20719

PMID:16385581

27. Dyer AH, Vahdatpour C, Sanfeliu A, Tropea D. The role of Insulin-Like Growth Factor 1 (IGF-1) in brain development, maturation and neuroplasticity. Neuroscience. 2016; 325:89-99.

https://doi.org/10.1016/i.neuroscience.2016.03.056 PMID:27038749

28. Colao A, Grasso LF, Giustina A, Melmed S, Chanson P, Pereira AM, Pivonello R. Acromegaly. Nat Rev Dis Primers. 2019; 5:20.

https://doi.org/10.1038/s41572-019-0071-6 PMID:30899019

29. Sievers C, Sämann PG, Dose T, Dimopoulou C, Spieler D, Roemmler J, Schopohl J, Mueller M, Schneider HJ, Czisch M, Pfister H, Stalla GK. Macroscopic brain architecture changes and white matter pathology in acromegaly: a clinicoradiological study. Pituitary. 2009; 12:177-85.

https://doi.org/10.1007/s11102-008-0143-1 PMID: 18836838

30. Pfefferbaum A, Mathalon DH, Sullivan EV, Rawles JM, Zipursky RB, Lim KO. A quantitative magnetic resonance imaging study of changes in brain morphology from infancy to late adulthood. Arch Neurol. 1994; 51:874-87.

https://doi.org/10.1001/archneur.1994.005402100460 12 PMID:8080387

31. Matsumae M, Kikinis R, Mórocz IA, Lorenzo AV, Sándor T, Albert MS, Black PM, Jolesz FA. Age-related changes in intracranial compartment volumes in normal adults assessed by magnetic resonance imaging. J Neurosurg. 1996; 84:982-91.

https://doi.org/10.3171/ins.1996.84.6.0982 PMID:8847593

32. Webb EA, Elliott L, Carlin D, Wilson M, Hall K, Netherton J, Reed J, Barrett TG, Salwani V, Clayden JD, Arlt W, Krone N, Peet AC, Wood AG. Quantitative Brain MRI in Congenital Adrenal Hyperplasia: In Vivo Assessment of the Cognitive and Structural Impact of Steroid Hormones. J Clin Endocrinol Metab. 2018; 103:1330-41.

https://doi.org/10.1210/ic.2017-01481

PMID:29165577 
33. Corre C, Friedel M, Vousden DA, Metcalf A, Spring S, Qiu LR, Lerch JP, Palmert MR. Separate effects of sex hormones and sex chromosomes on brain structure and function revealed by high-resolution magnetic resonance imaging and spatial navigation assessment of the Four Core Genotype mouse model. Brain Struct Funct. 2016; 221:997-1016.

https://doi.org/10.1007/s00429-014-0952-0

PMID:25445841

34. Bianchi VE, Locatelli V, Rizzi L. Neurotrophic and Neuroregenerative Effects of GH/IGF1. Int J Mol Sci. 2017; 18: 2441.

https://doi.org/10.3390/ijms18112441

PMID:29149058

35. Farokhian F, Yang C, Beheshti I, Matsuda H, Wu S. AgeRelated Gray and White Matter Changes in Normal Adult Brains. Aging Dis. 2017; 8:899-909.

https://doi.org/10.14336/AD.2017.0502

PMID:29344423

36. Muller AP, Fernandez AM, Haas C, Zimmer E, Portela LV, Torres-Aleman I. Reduced brain insulin-like growth factor I function during aging. Mol Cell Neurosci. 2012; 49:9-12.

https://doi.org/10.1016/i.mcn.2011.07.008

PMID:21807098

37. Hochberg Z, Phillip M, Youdim MB, Amit T. Regulation of the growth hormone $(\mathrm{GH})$ receptor and $\mathrm{GH}$-binding protein by $\mathrm{GH}$ pulsatility. Metabolism. 1993; 42:1617-23.

https://doi.org/10.1016/0026-0495(93)90159-L

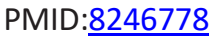

38. De Keyser J, Wilczak N, De Backer JP, Herroelen L, Vauquelin G. Insulin-like growth factor-I receptors in human brain and pituitary gland: an autoradiographic study. Synapse. 1994; 17:196-202.

https://doi.org/10.1002/syn.890170309

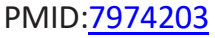

39. Carro E, Trejo JL, Gomez-Isla T, LeRoith D, TorresAleman I. Serum insulin-like growth factor I regulates brain amyloid-beta levels. Nat Med. 2002; 8:1390-7. https://doi.org/10.1038/nm793

PMID:12415260

40. George C, Gontier G, Lacube P, François JC, Holzenberger $M$, Aïd S. The Alzheimer's disease transcriptome mimics the neuroprotective signature of IGF-1 receptor-deficient neurons. Brain. 2017; 140:2012-27.

https://doi.org/10.1093/brain/awx132

PMID:28595357

41. Saccà F, Quarantelli $M$, Rinaldi C, Tucci T, Piro R, Perrotta G, Carotenuto B, Marsili A, Palma V, De Michele G, Brunetti A, Brescia Morra V, Filla A,
Salvatore M. A randomized controlled clinical trial of growth hormone in amyotrophic lateral sclerosis: clinical, neuroimaging, and hormonal results. J Neurol. 2012; 259:132-8.

https://doi.org/10.1007/s00415-011-6146-2

PMID:21706151

42. Kaspar BK, Frost LM, Christian L, Umapathi P, Gage FH. Synergy of insulin-like growth factor-1 and exercise in amyotrophic lateral sclerosis. Ann Neurol. 2005; 57:649-55.

https://doi.org/10.1002/ana.20451

PMID:15852403

43. Moon CM, Kim BC, Jeong GW. Effects of donepezil on brain morphometric and metabolic changes in patients with Alzheimer's disease: A DARTEL-based VBM and (1)H-MRS. Magn Reson Imaging. 2016; 34:1008-16. https://doi.org/10.1016/i.mri.2016.04.025 PMID:27131829

44. Matsuda H. MRI morphometry in Alzheimer's disease. Ageing Res Rev. 2016; 30:17-24. https://doi.org/10.1016/j.arr.2016.01.003 PMID:26812213

45. Christidi F, Karavasilis E, Riederer F, Zalonis I, Ferentinos $P$, Velonakis $G$, Xirou S, Rentzos $M$, Argiropoulos G, Zouvelou V, Zambelis T, Athanasakos A, Toulas $P$, et al. Gray matter and white matter changes in non-demented amyotrophic lateral sclerosis patients with or without cognitive impairment: A combined voxel-based morphometry and tract-based spatial statistics whole-brain analysis. Brain Imaging Behav. 2018; 12:547-63.

https://doi.org/10.1007/s11682-017-9722-y PMID:28425061

46. Stewart HJ, Bradke F, Tabernero A, Morrell D, Jessen KR, Mirsky R. Regulation of rat Schwann cell Po expression and DNA synthesis by insulin-like growth factors in vitro. Eur J Neurosci. 1996; 8:553-64. https://doi.org/10.1111/i.1460-9568.1996.tb01240.x PMID:8963447

47. Kanje M, Skottner A, Lundborg G. Effects of growth hormone treatment on the regeneration of rat sciatic nerve. Brain Res. 1988; 475:254-58. https://doi.org/10.1016/0006-8993(88)90613-0 PMID: $\underline{3214734}$

48. Devesa P, Gelabert M, Gonźlez-Mosquera T, Gallego R, Relova JL, Devesa J, Arce VM. Growth hormone treatment enhances the functional recovery of sciatic nerves after transection and repair. Muscle Nerve. 2012; 45:385-92. https://doi.org/10.1002/mus.22303 PMID:22334173

49. Feeney C, Sharp DJ, Hellyer PJ, Jolly AE, Cole JH, Scott G, Baxter D, Jilka S, Ross E, Ham TE, Jenkins PO, Li LM, 
Gorgoraptis N, et al. Serum insulin-like growth factor-I levels are associated with improved white matter recovery after traumatic brain injury. Ann Neurol.
2017; 82:30-43.

https://doi.org/10.1002/ana.24971

PMID:28574152 


\section{SUPPLEMENTARY MATERIALS}

Supplementary Figures
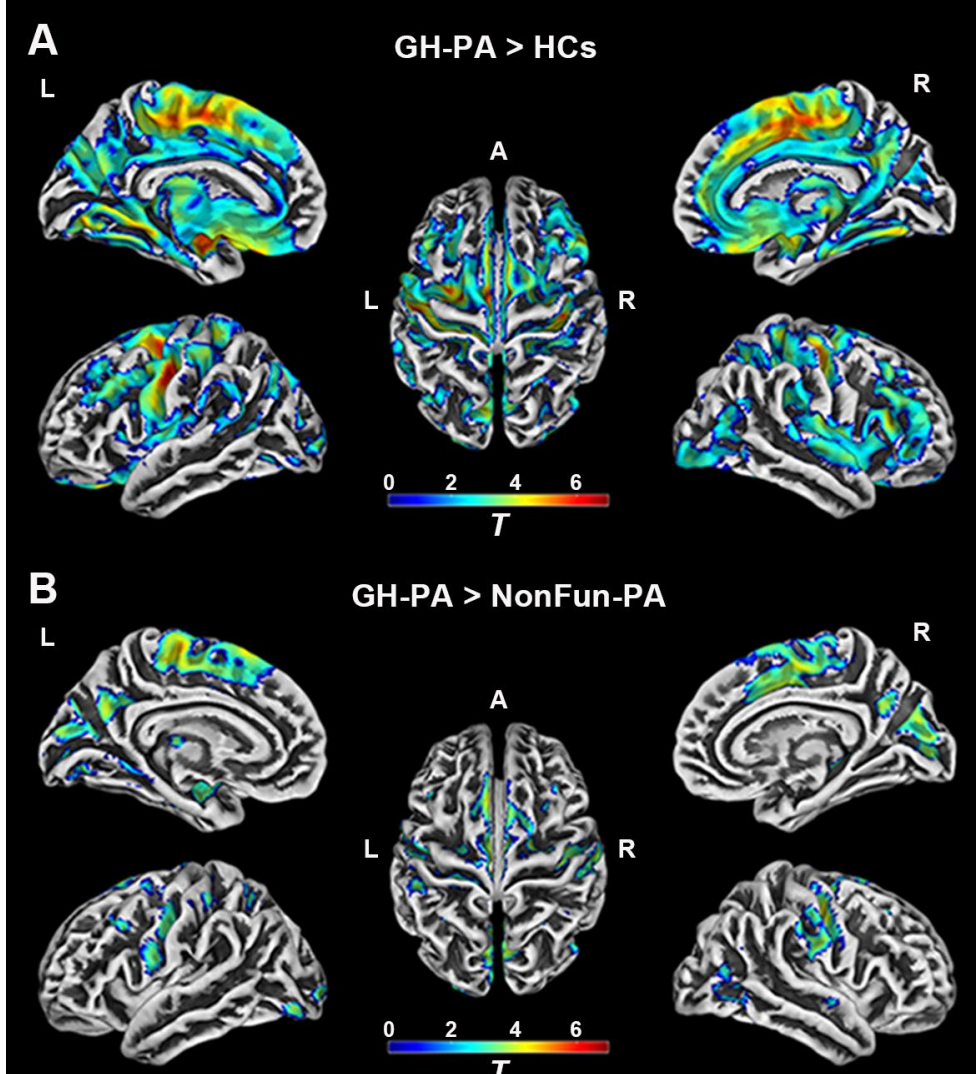

GH-PA > NonFun-PA
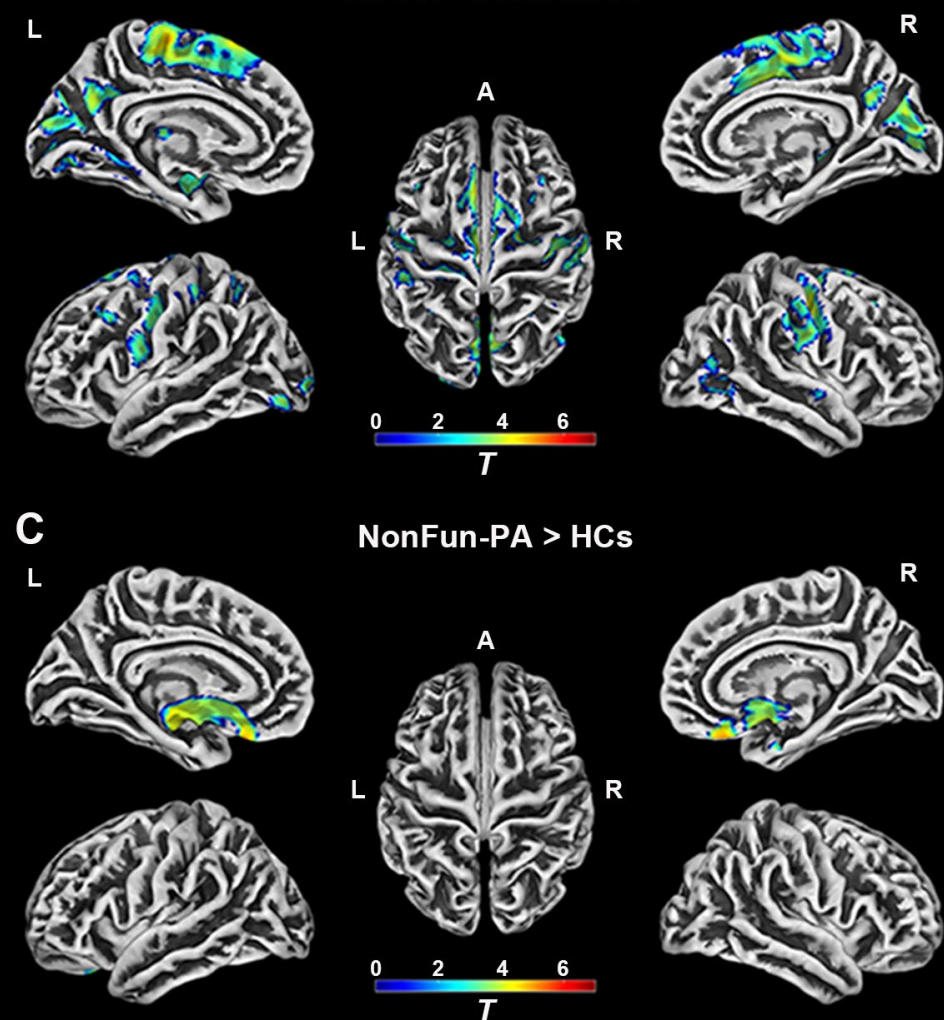

NonFun-PA > HCs

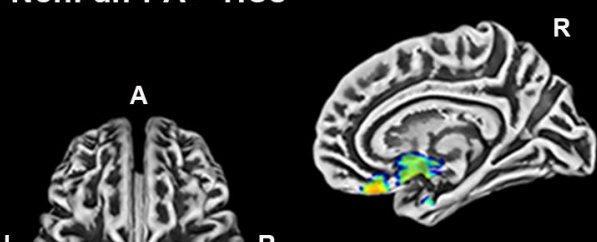

L
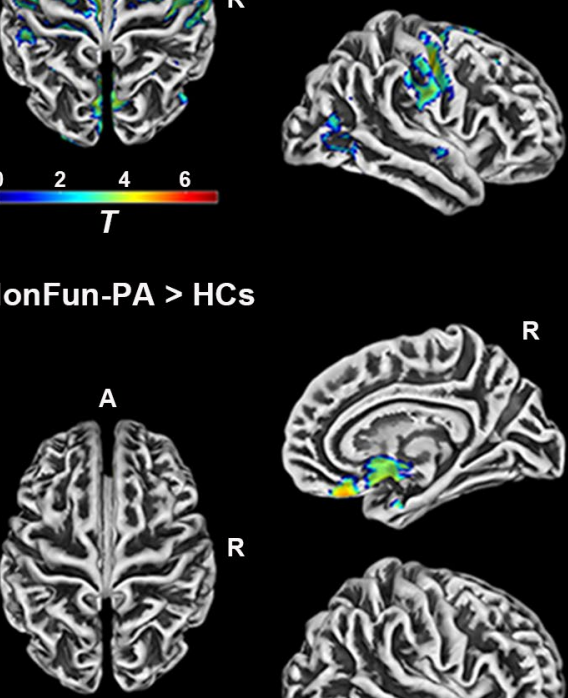

\begin{tabular}{llll}
0 & 2 & 4 & 6 \\
\hline
\end{tabular}

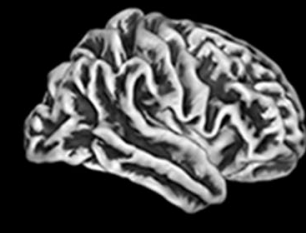

Supplementary Figure 1. GM regional differences. VBM analysis showing the extensive regions of GMV increases in GH-PA compared to HCs (A) and NonFun-PA (B) (FDR corrected, $p<0.05$, cluster size $>333$ ). VBM analysis showing GMV alteration in NonFun-PA compared to HCs (C) (FDR corrected, $p<0.05$, cluster size $>333$ ). 


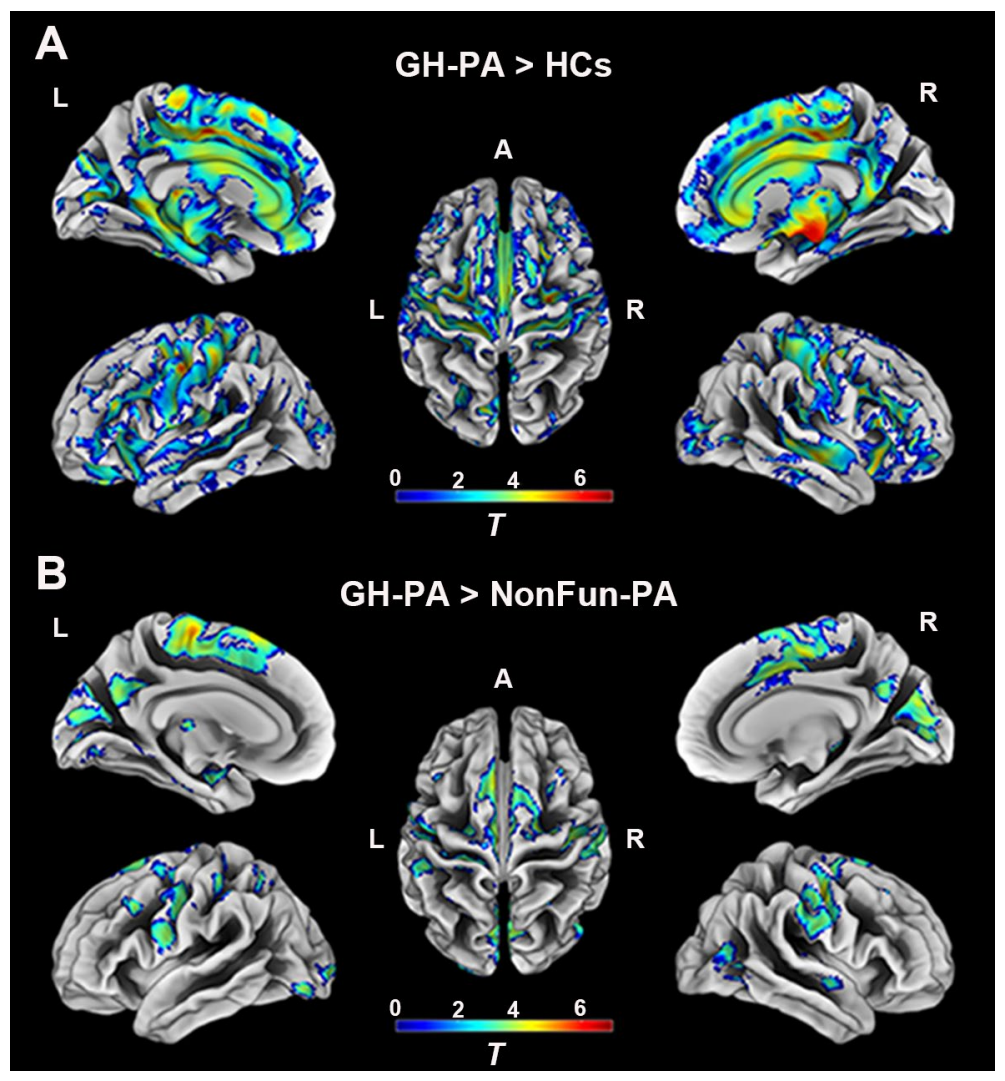

Supplementary Figure 2. WM regional differences. VBM analysis showing the extensive regions of WMV increases in GH-PA compared to HCs (A) and NonFun-PA (B) (FDR corrected, $\mathrm{p}<0.05$, cluster size $>333$ ).

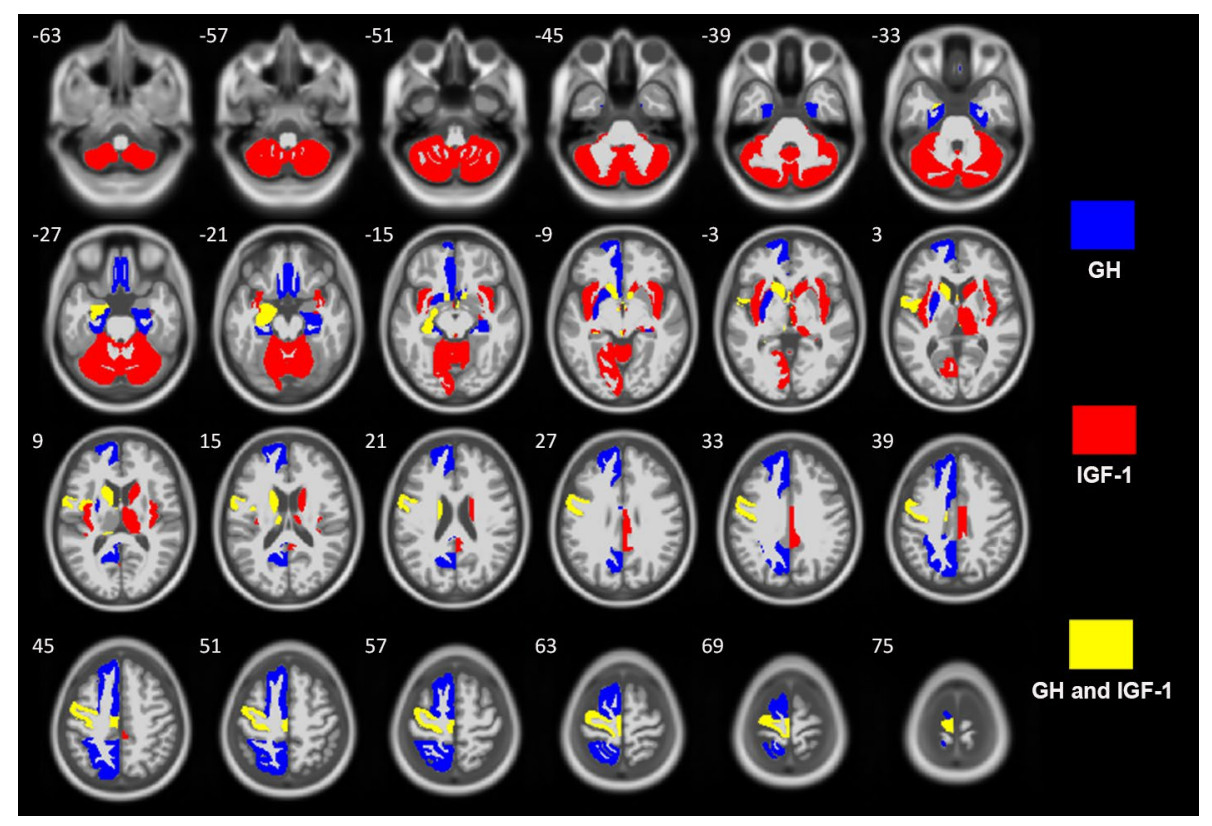

Supplementary Figure 3. Correlation analysis between GH/IGF-1 and gray matter volume (GMV) of brain regions (Hammers' atlas) in GH-PA patients. Blue represents brain regions volumes that are positively correlated with serum GH levels; red represents brain region volumes that are positively correlateed with serum IGF-1 levels; yellow represents brain region volumes that are simultaneously positively correlated with serum GH levels and IGF-1 levels (Supplementary Table 2). Significance was determined by $p<0.05$. 


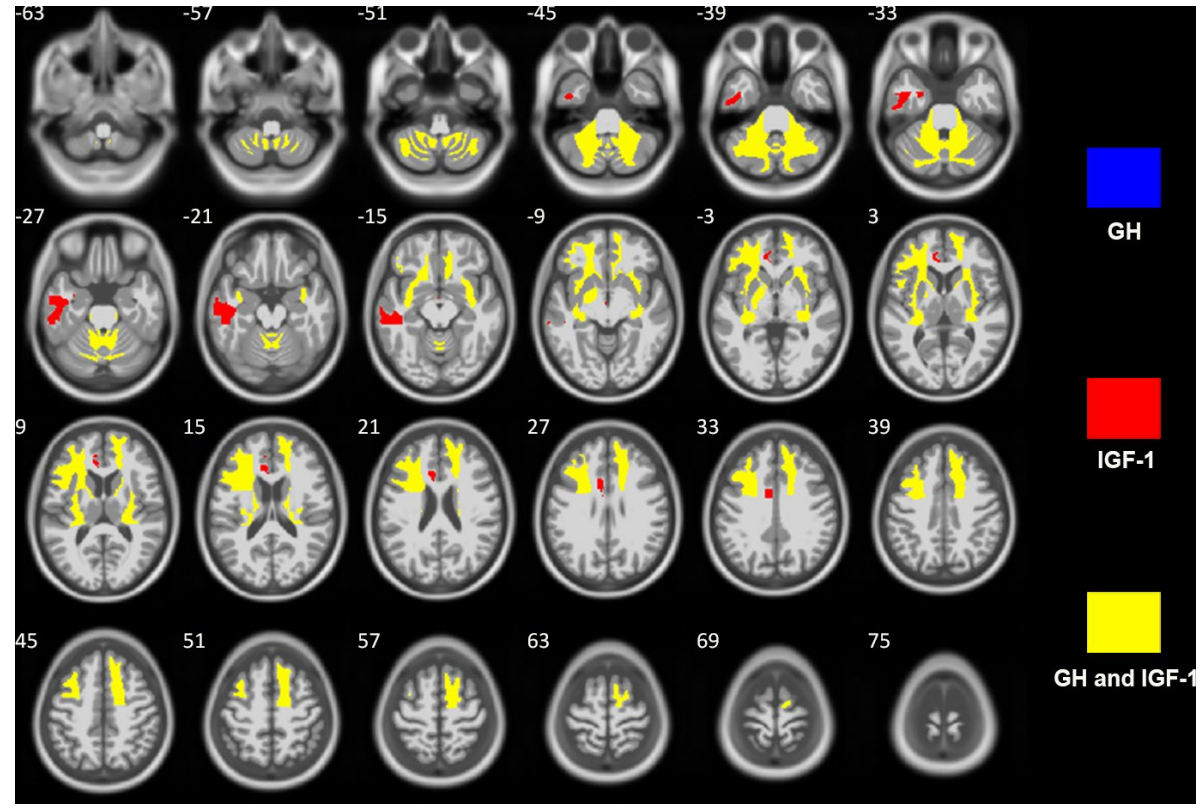

Supplementary Figure 4. Correlation analysis between GH/IGF-1 and white matter volume (WMV) of brain regions (Hammers' atlas) in GH-PA patients. Blue represents brain region volumes that are positively correlated with serum GH levels; red represents brain regions volumes that are positively correlated with serum IGF-1 levels; yellow represents brain region volumes that are simultaneously positively correlated with serum GH levels and IGF-1 levels (Supplementary Table 3). Significance was determined by $p<0.05$. 


\section{Supplementary Tables}

Supplementary Table 1. Specific brain regions of gray matter (GM) volume and white matter (WM) volume affected by serum GH/IGF-1 level in patients compared with controls (Hammers' atlas).

\begin{tabular}{|c|c|c|c|}
\hline Abnormal brain region (GM) & T value & Abnormal brain region (WM) & T value \\
\hline lHip & 2.10 & rHip & 2.11 \\
\hline rHip & 2.52 & 1AntMedTeLo & 2.26 \\
\hline 1Amy & 4.17 & 1AntLatTeLo & 2.73 \\
\hline rAmy & 2.95 & 1AmbParHipGy & 3.64 \\
\hline 1AmbParHipGy & 2.76 & rAmbParHipGy & 2.25 \\
\hline rAmbParHipGy & 3.02 & 1SupTemGy & 3.57 \\
\hline 1SupTemGy & 2.51 & rSupTemGy & 3.51 \\
\hline rSupTemGy & 3.20 & IInfMidTemGy & 3.30 \\
\hline lCbe & 3.58 & rInfMidTemGy & 3.37 \\
\hline $\mathrm{rCbe}$ & 4.26 & lCbe & 4.05 \\
\hline 1Bst & 5.16 & rCbe & 3.48 \\
\hline rBst & 5.22 & lBst & 5.25 \\
\hline IIns & 4.06 & rBst & 5.40 \\
\hline rIns & 4.23 & IIns & 5.19 \\
\hline ILatOcLo & 2.03 & rIns & 4.55 \\
\hline rLatOcLo & 2.68 & 1LatOcLo & 2.84 \\
\hline 1AntCinGy & 3.30 & rLatOcLo & 2.95 \\
\hline rAntCinGy & 3.12 & 1AntCinGy & 2.89 \\
\hline 1PosCinGy & 3.83 & rAntCinGy & 3.15 \\
\hline rPosCinGy & 3.97 & 1PosCinGy & 3.55 \\
\hline 1MidFroGy & 3.68 & rPosCinGy & 3.74 \\
\hline rMidFroGy & 3.45 & lMidFroGy & 5.49 \\
\hline 1PosTeLo & 3.10 & rMidFroGy & 4.86 \\
\hline rPosTeLo & 3.88 & 1PosTeLo & 4.61 \\
\hline IInfLatPaLo & 2.90 & rPosTeLo & 4.81 \\
\hline rInfLatPaLo & 2.40 & IInfLatPaLo & 3.67 \\
\hline 1CauNuc & 3.60 & rInfLatPaLo & 3.72 \\
\hline rCauNuc & 3.32 & 1CauNuc & 5.64 \\
\hline 1AccNuc & 4.15 & rCauNuc & 5.83 \\
\hline rAccNuc & 4.70 & lPut & 3.79 \\
\hline lPut & 3.66 & rPut & 3.31 \\
\hline rPut & 3.49 & ITha & 4.26 \\
\hline 1Tha & 3.55 & rTha & 4.64 \\
\hline rTha & 3.50 & lPal & 5.43 \\
\hline 1LatTemVen & 4.51 & $\mathrm{rPal}$ & 5.69 \\
\hline rLatTemVen & 4.36 & $1 \mathrm{CC}$ & 3.39 \\
\hline 13thVen & 4.02 & $\mathrm{rCC}$ & 3.78 \\
\hline r3thVen & 3.69 & ILatTemVen & 4.01 \\
\hline 1PrcGy & 5.77 & rLatTemVen & 3.93 \\
\hline
\end{tabular}




\begin{tabular}{lccc} 
rPrcGy & 4.73 & IPrcGy & 6.75 \\
IRecGy & 4.71 & rPrcGy & 5.07 \\
rRecGy & 3.51 & lRecGy & 2.39 \\
IInfFroGy & 2.38 & rRecGy & 2.49 \\
rInfFroGy & 3.60 & 1OrbFroGy & 2.60 \\
ISupFroGy & 4.02 & rOrbFroGy & 2.77 \\
rSupFroGy & 4.02 & InfFroGy & 3.93 \\
IPoCGy & 4.01 & rInfFroGy & 4.26 \\
rPoCGy & 3.73 & 1 SupFroGy & 5.25 \\
ISupParGy & 3.26 & rSupFroGy & 4.82 \\
rSupParGy & 3.36 & PPoCGy & 5.94 \\
ILinGy & 3.61 & rPoCGy & 6.24 \\
rLinGy & 2.06 & 1 SupParGy & 4.01 \\
ICun & 3.10 & rSupParGy & 5.00 \\
rCun & 2.07 & 1 Cun & 3.02 \\
\hline
\end{tabular}

Supplementary Table 2. Specific gray matter (GM) regions correlated with serum GH/IGF-1 level in patients (Hammers' atlas).

\begin{tabular}{|c|c|c|c|c|c|}
\hline GH Correlation & r value & p-value & IGF-1 Correlation & r value & p value \\
\hline Grey Matter & & & Grey Matter & & \\
\hline 1Hip & 0.42 & $<0.05$ & 1Hip & 0.36 & $<0.05$ \\
\hline rHip & 0.31 & $<0.05$ & 1Amy & 0.37 & $<0.05$ \\
\hline 1Amy & 0.43 & $<0.05$ & $1 \mathrm{Cbe}$ & 0.32 & $<0.05$ \\
\hline 1AmbParHipGy & 0.43 & $<0.05$ & rCbe & 0.31 & $<0.05$ \\
\hline rAmbParHipGy & 0.42 & $<0.05$ & 1Bst & 0.43 & $<0.05$ \\
\hline 1Bst & 0.34 & $<0.05$ & rBst & 0.37 & $<0.05$ \\
\hline lCauNuc & 0.29 & $<0.05$ & IIns & 0.34 & $<0.05$ \\
\hline 1AccNuc & 0.64 & $<0.05$ & rIns & 0.36 & $<0.05$ \\
\hline rAccNuc & 0.62 & $<0.05$ & rPosCinGy & 0.30 & $<0.05$ \\
\hline lPut & 0.38 & $<0.05$ & 1CauNuc & 0.43 & $<0.05$ \\
\hline $1 C C$ & 0.32 & $<0.05$ & rCauNuc & 0.37 & $<0.05$ \\
\hline 1LatTemVen & 0.30 & $<0.05$ & 1AccNuc & 0.34 & $<0.05$ \\
\hline r3thVen & 0.37 & $<0.05$ & rAccNuc & 0.39 & $<0.05$ \\
\hline 1PrcGy & 0.32 & $<0.05$ & rTha & 0.35 & $<0.05$ \\
\hline 1RecGy & 0.44 & $<0.05$ & 1LatTemVen & 0.31 & $<0.05$ \\
\hline rRecGy & 0.42 & $<0.05$ & rLatTemVen & 0.32 & $<0.05$ \\
\hline 1SupFroGy & 0.29 & $<0.05$ & 13thVen & 0.36 & $<0.05$ \\
\hline \multirow[t]{2}{*}{ rSupFroGy } & 0.32 & $<0.05$ & r3thVen & 0.41 & $<0.05$ \\
\hline & & & 1PrcGy & 0.35 & $<0.05$ \\
\hline
\end{tabular}


Supplementary Table 3. Specific white matter (WM) regions correlated with serum GH/IGF-1 level in patients (Hammers' atlas).

\begin{tabular}{lccccc}
\hline GH Correlation & r value & p value & IGF-1 Correlation & r value & p value \\
\hline White Matter & & & White Matter & & \\
lAmy & 0.36 & $<0.05$ & ICbe & 0.32 & $<0.05$ \\
IInfMidTemGy & 0.32 & $<0.05$ & rCbe & 0.37 & $<0.05$ \\
IAntCinGy & 0.30 & $<0.05$ & IIns & 0.32 & $<0.05$ \\
13thVen & 0.33 & $<0.05$ & rIns & 0.29 & $<0.05$ \\
rPrcGy & 0.32 & $<0.05$ & IMidFroGy & 0.32 & $<0.05$ \\
& & & ICauNuc & 0.30 & $<0.05$ \\
& & rCauNuc & 0.29 & $<0.05$ \\
& & IPal & 0.32 & $<0.05$ \\
& & IInfFroGy & 0.29 & $<0.05$ \\
\end{tabular}

